\title{
Hyperandrogenism in female athletes with
}

\section{functional hypothalamic amenorrhea: a distinct phenotype}

\author{
Asma Javed' \\ Rahul Kashyap ${ }^{2}$ \\ Aida N Lteif' \\ 'Pediatric and Adolescent Medicine, \\ Division of Pediatric Endocrinology \\ Mayo Clinic, Rochester, MN, USA; \\ ${ }^{2}$ Department of Anesthesia and \\ Critical Care Medicine, Mayo Clinic, \\ Rochester, MN, USA
}

This article was published in the following Dove Press journal:

International Journal of Women's Health

13 January 2015

Number of times this article has been viewed

Objective: To compare the reproductive, metabolic, and skeletal profiles of young athletic women with functional hypothalamic amenorrhea (FHA) as well as clinical or biochemical hyperandrogenism (FHA-EX+HA) with body mass index matched women with FHA due to exercise (FHA-EX) or anorexia nervosa (FHA-AN) alone.

Design: Retrospective cohort study.

Setting: Tertiary care teaching hospital.

Population: Adolescents and young women, 15-30 years of age, diagnosed with FHA along with concurrent signs of hyperandrogenism $(n=22)$ and body mass index matched control groups consisting of 22 women in each group of FHA-EX and FHA-AN.

Main outcomes: 1) Reproductive hormone profile: luteinizing hormone (LH), follicle stimulating hormone (FSH), total testosterone, pelvic ultrasound features. 2) Metabolic function and skeletal health markers: fasting glucose, cholesterol, number of stress fractures and bone mineral density as assessed by spine dual-energy X-ray absorptiometry $z$ scores.

Results: FHA-EX+HA group was older at diagnosis compared to the other groups with a median (interquartile range [IQR]) age of $22(18.75-25.25)$ years versus (vs) 17.5 $(15.75-19)$ for FHA-EX; $(P<0.01)$ and $18(16-22.25)$ years for FHA-AN $(P=0.01)$. There were no differences among the groups based on number of hours of exercise per week, type of physical activity or duration of amenorrhea. Median (IQR) LH/FSH ratio was higher in FHA-EX+HA than both other groups, $1.44(1.03-1.77)$ vs $0.50(0.20-0.94)$ for FHA-EX and $0.67(0.51-0.87)$ for FHA-AN ( $P<0.01$ for both). Total testosterone concentrations were not different among the groups. Median (IQR) fasting serum glucose concentration was higher in FHA-EX+HA vs FHA-EX, $88.5 \mathrm{mg} / \mathrm{dL}(82.8-90 \mathrm{mg} / \mathrm{dL})$ vs $83.5 \mathrm{mg} / \mathrm{dL}(78.8-86.3 \mathrm{mg} / \mathrm{dL})(P=0.01)$ but not different from FHA-AN $(P=0.31)$. Percentage of women with stress fractures was lower in FHA-EX+HA (4.5\%) as compared to both FHA-EX $(27.3 \%)$ and FHA-AN (50\%); $P=0.04$ and 0.01 respectively. The LH/FSH ratio was weakly positively associated with serum glucose (adjusted $r^{2}=0.102 ; P=0.01$ ) as well as with dual-energy X-ray absorptiometry spine score (adjusted $r^{2}=0.191 ; P=0.04$ ) in the entire cohort.

Conclusion: In a small cohort of female athletes with hyperandrogenism, a distinct reproductive hormone profile consisting of higher LH to FHS ratio may be associated with adverse metabolic health markers but improved skeletal health.

Keywords: functional hypothalamic amenorrhea, hyperandrogenism, polycystic ovary syndrome, young athletes

Correspondence: Aida N Lteif

Pediatric and Adolescent Medicine,

Division of Pediatric Endocrinology, Mayo

Clinic, 200 First Street SW, Rochester

55905, MN, USA

Tel + I 5072843300

$\mathrm{Fax}+\mathrm{I} 5072840727$

Email Iteif.aida@mayo.edu

\section{Introduction}

Adolescents and young women frequently suffer from menstrual dysfunction. Functional hypothalamic amenorrhea (FHA) and hyperandrogenism syndromes such as polycystic ovary syndrome (PCOS) are the most common causes of menstrual 
irregularity in this age group. ${ }^{1,2}$ FHA is a condition characterized by the absence of menses due to the suppression of the hypothalamic-pituitary-ovarian axis, in which no anatomical or organic disease is identified. ${ }^{3}$ Adolescents or young women with this condition typically present with amenorrhea of 6 months' duration or longer. ${ }^{4}$ This condition may be difficult to differentiate from immaturity of the hypothalamicpituitary-ovarian axis during the initial post-menarchal years in adolescents but even during this time, menstrual cycles in adolescents typically are no longer than 45 days. ${ }^{5}$ FHA in this population can occur secondary to disordered eating and/or excessive exercise. On the other hand, PCOS is a complex endocrinopathy with marked heterogeneity in clinical manifestations. Often associated with obesity, PCOS affects $4 \%-10 \%$ of women of reproductive age ${ }^{6-10}$ and comprises a combination of hyperandrogenism, ovulatory dysfunction, and morphologically polycystic ovaries. ${ }^{1-14}$ PCOS is often associated with concomitant abnormalities of insulin resistance with or without compensatory hyperinsulinemia independent of body weight, ${ }^{15-18}$ dyslipidemia, ${ }^{19}$ and subfertility. ${ }^{18}$

There is emerging evidence of certain subsets of athletic females suffering from menstrual dysfunction with clinical features and hormonal profiles suggestive of androgen excess, in some cases meeting criteria for PCOS, challenging the contemporary concept that reproductive dysfunction in athletes is due to chronic energy deficiency alone. ${ }^{1,20}$ Varying athletic disciplines, based on the intensity of exercise and energy deficit incurred, may impact gonadotropin-releasing hormone pulsatility in different ways. ${ }^{21}$ In fact, a systematic review assessing observational studies as well as randomized clinical trials in 2009 reported that although data are still scarce, there is a suggestion that hyperandrogenism, such as that in PCOS, may likely be associated with oligomenorrhea in exercising women, and may not always represent hypothalamic inhibition due to low energy availability. In sports emphasizing leanness, nutritional restriction may be an important causal factor in reproductive dysfunction but a distinct hormonal profile characterized by hyperandrogenism rather than hypoestrogenism has been described in sports emphasizing strength over leanness. ${ }^{21}$

It has also been postulated that in subjects with FHA and concurrent features of PCOS, some athletic advantage may be afforded. ${ }^{22}$

PCOS is often associated with adverse metabolic profile although it is not known if the metabolic milieu of athletes with hyperandrogenism is inferior to that of athletes with FHA alone.
The primary aim of our study was to compare reproductive hormone profile among adolescents and young women with concurrent diagnoses of exercise induced FHA as well as clinical or biochemical hyperandrogenism (FHA-EX+HA) to athletes with FHA only, either due to excessive exercise (FHA-EX) or anorexia nervosa (FHA-AN). Secondarily, we compared the metabolic and skeletal profiles of females in each group and explored the association, if any, between the reproductive and skeletal or metabolic profiles.

\section{Methods}

This was a retrospective review of medical records of female adolescents and young women seen at Mayo Clinic, Rochester, MN between 2004-2013, for female reproductive health disorders including FHA and PCOS.

\section{Participants}

After receiving the approval of the Institutional Review Board at Mayo Clinic, Rochester, MN, adolescents and young women aged 15-30 years with concurrent diagnoses of FHA and hyperandrogenism (FHA-EX+HA) or FHA-EX or FHA-AN were identified using both a free text search in clinical notes in addition to coding and billing data. Identification of the cohorts was performed using the Mayo Clinic Life Sciences System, which is a complete clinical data repository containing patient demographics; diagnoses; hospital-, laboratory-, and clinical notes; pathology; and billing data obtained from multiple clinical and hospital source systems within Mayo Clinic. Data in the Mayo Clinic Life Sciences System was accessed via the Data Discovery and Query Builder toolset, consisting of a web-based application that utilizes a unique text search engine and provides the capability to rapidly search for specific words and phrases within unstructured text documents such as clinical notes. ${ }^{23}$ All adolescents and young women seen within a 10 year period (January 2004 to December 2013) with the International Classification of Diseases, Ninth Revision (ICD-9) code for PCOS (256.4) and amenorrhea (626.0) supplemented with text word search terms such as "female athlete triad", or "exercise induced menstrual dysfunction", or "exercise induced amenorrhea", or "functional hypothalamic amenorrhea", or "menstrual irregularity due to exercise" and "hyperandrogenism" or "excess androgens" were identified for Group 1 (FHAEX+HA). Exclusion terms of "eating disorder", "disordered eating", "anorexia nervosa", "bulimia nervosa", were also applied in order to not include females with disordered eating in Group 1. The same text search terms of "female athlete triad", "exercise induced menstrual dysfunction", 
"exercise induced amenorrhea", "functional hypothalamic amenorrhea", "menstrual irregularity due to exercise" with negation of "PCOS", "hyperandrogenism", "excess androgens" and "eating disorder", "disordered eating", "anorexia nervosa", "bulimia nervosa", were used to identify Group 2 (FHA-EX). This cohort was body mass index (BMI) matched with Group 1. For identification of Group 3 (FHA-AN), search terms of "eating disorder", "disordered eating", "anorexia nervosa", "bulimia nervosa", along with "menstrual dysfunction", "functional hypothalamic amenorrhea", "amenorrhea", "oligomenorrhea", "menstrual irregularity due to eating disorder", "menstrual irregularity due to disordered eating", "menstrual irregularity due to anorexia nervosa" were used. This group was also BMI matched with Group 1.

\section{Definitions}

Subjects in the FHA-EX+HA group were identified if they had a diagnosis of FHA due to excessive exercise but also had either clinical or biochemical evidence of hyperandrogenism. Rotterdam criteria were used to diagnose PCOS, requiring the presence of at least two of the three criteria of: 1) clinical or biochemical evidence of hyperandrogenism, 2) oligo- or anovulation (defined as less than nine periods per year), and 3) polycystic ovarian morphology (PCOM). During chart review, we identified girls who also met the National Institutes of Health criteria for PCOS requiring the presence of both oligo-ovulation and hyperandrogenism. Determination of PCOM was based on presence of 12 or more 2-9 $\mathrm{mm}$ follicles in each ovary or ovarian volume $>10 \mathrm{~mL}$ as defined by Rotterdam criteria. ${ }^{24}$ FHA due to exercise was defined as absence of menstrual cycles for 6 or more months in an otherwise healthy female exercising at least 8 hours/ week without any chronic severe illness. ${ }^{3}$ Type of physical activity was divided into aerobic, anaerobic or both. FHA due to anorexia nervosa was defined as absence of menstrual cycles for 6 or more months in a female with previously regular menstrual cycles with a diagnosis of anorexia nervosa made by a psychiatrist or psychologist.

Subjects with Turner's syndrome, premature ovarian failure, untreated hypothyroidism, hyperprolactinemia, congenital adrenal hyperplasia, Cushing's syndrome, pregnancy, androgen-secreting tumors, subjects on any form of hormonal contraception or a condition as the cause of hyperandrogenism were excluded.

\section{Procedures and measures}

We employed a standardized abstraction form for data collection. For each medical record, we identified the first visit in which the diagnosis of FHA was made. We collected information regarding age at diagnosis, age at menarche, diagnosis of PCOS or hyperandrogenism (Group 1), BMI, systolic and diastolic blood pressure, number of hours of exercise per week, type of physical activity, number of fractures (stress and long bone fractures), pattern of menstrual dysfunction (primary amenorrhea, secondary amenorrhea or oligomenorrhea). Biochemical measurements collected, where available, included LH, FSH, total and free testosterone, calcium, phosphorus, alkaline phosphatase, fasting glucose, total cholesterol, triglycerides, low density lipoprotein and high density lipoprotein. Radiographic information if available was obtained on duel-energy X-ray absorptiometry (DXA) spine and hip $z$ scores as well as pelvic ultrasonography.

All biochemical laboratory measures were obtained in the Mayo Clinic Laboratories using standard automated colorimetric enzymatic assays (Roche Diagnostics, Indianapolis, IN, USA) with the exception of testosterone, which was measured using high-throughput liquid chromatography (Hamilton Robotics Inc., Reno, NV, USA), and glucose, which was measured using a photometric rate reaction (Roche Diagnostics). Fasting glucose was obtained using a standard minimum 12 hours of fasting, which was verified on all samples.

\section{Statistical analyses}

We compared the medians and interquartile ranges (IQRs) of clinical and biochemical characteristics between FHA-EX+ HA and FHA-EX and FHA-AN using Wilcoxon test. Categorical variables were compared using chi-square analysis. Linear regression models were created in a stepwise fashion using JMP 9.0.1 (SAS Institute Inc., Cary, NC, USA) to identify associations of luteinizing hormone ( $\mathrm{LH}) /$ follicle stimulating hormone (FSH) ratio with skeletal and metabolic parameters.

\section{Results \\ Cohort derivation}

\section{Group I: FHA due to exercise with} hyperandrogenism (FHA-EX+HA)

Between January 2004 and December 2013, a total of 58 women were identified, who were aged between 15-30 years with a concurrent electronic documentation of both exercise related FHA and clinical or biochemical hyperandrogenism. We determined by individual chart if the subject met criteria for PCOS (Figure 1). After exclusion of subjects with other chronic illnesses and women on hormonal medication use at the time of diagnosis, a total 


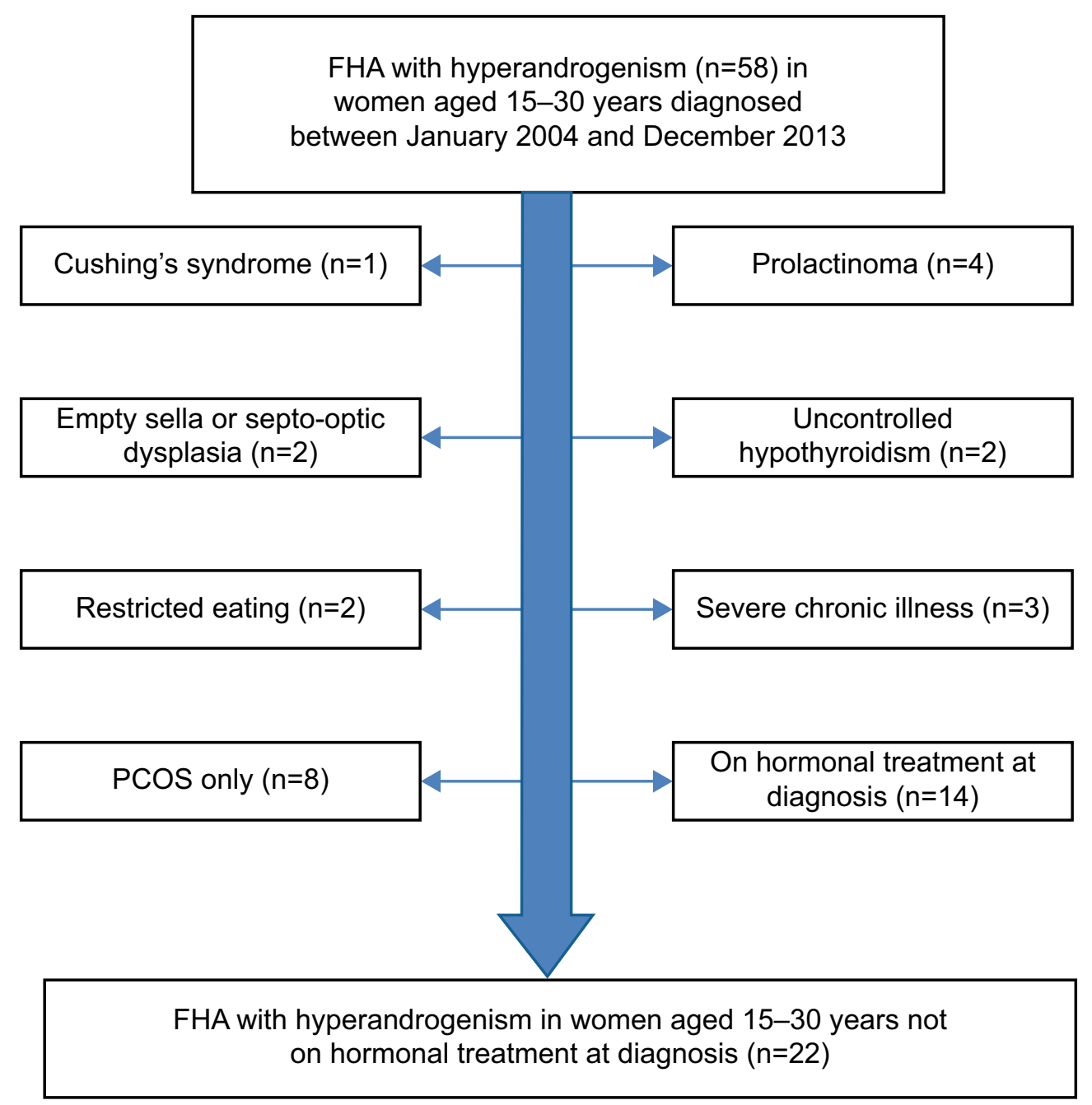

Figure I Flow diagram depicting selection of FHA-EX+HA cohort.

Abbreviations: FHA, functional hypothalamic amenorrhea; PCOS, polycystic ovary syndrome.

of 22 subjects with FHA due to excessive exercise and evidence of hyperandrogenism at the time of presentation were included in the analysis.

\section{Group 2: FHA due to excessive exercise} without hyperandrogenism (FHA-EX)

For every case of FHA-EX+HA, women aged 15-30 years with FHA due to excessive exercise but no evidence of disordered eating, not on any hormonal treatment at diagnosis and with no features of hyperandrogenism were BMI matched (within $1 \mathrm{~kg} / \mathrm{m}^{2}$ ) with the corresponding case of FHA-EX+HA. Exclusion criteria were: 1) presence of any restricted or disordered eating, 2) anorexia nervosa or bulimia nervosa, 3) clinical and/or biochemical hyperandrogenism (ie, presence of hirsutism or Ferriman Gallwey score $>6$ or serum total testosterone $>60 \mathrm{ng} / \mathrm{dL}$ ), 4) hyperprolactinemia or 5) untreated hypothyroidism.

\section{Group 3: FHA due to anorexia nervosa} without hyperandrogenism (FHA-AN)

For every case of FHA-EX+HA, women aged 15-30 years with FHA due to anorexia nervosa (FHA-AN), not on any hormonal treatment at diagnosis and no features of hyperandrogenism (as described in the Methods section), were BMI matched (within $1 \mathrm{~kg} / \mathrm{m}^{2}$ ) with the corresponding case of FHA-EX+HA.

\section{Clinical and anthropometric characteristics}

FHA-EX+HA subjects were older at diagnosis compared to the other groups with a median (IQR) age of $22(18.8-25.3)$ years versus (vs) $17.5(15.8-19)$ for FHA-EX $(P<0.01)$ and 18 (16-22.3) for FHA-AN; $P=0.01$. There was no difference between the two groups based on ethnicity, BMI or age at menarche (Table 1). Median (IQR) reported weight loss (kg) 
Table I Baseline characteristics of all three groups

\begin{tabular}{|c|c|c|c|c|c|}
\hline Characteristic & $\begin{array}{l}\text { Group I } \\
\text { FHA-EX+HA } \\
(\mathbf{n}=\mathbf{2 2})\end{array}$ & $\begin{array}{l}\text { Group } 2 \\
\text { FHA-EX } \\
(n=22)\end{array}$ & $\begin{array}{l}\text { Group } 3 \\
\text { FHA-AN } \\
(n=22)\end{array}$ & $\begin{array}{l}P \text {-value } \\
\text { group I } \\
\text { and } 2\end{array}$ & $\begin{array}{l}P \text {-value } \\
\text { group I } \\
\text { and } 3 \\
\end{array}$ \\
\hline Age at diagnosis, in years, median (IQR) & $22(\mid 8.7-25)$ & $17.5(15.7-19)$ & $18(16-22)$ & $<0.01 *$ & $0.01 *$ \\
\hline BMI $\left(\mathrm{kg} / \mathrm{m}^{2}\right)$, median (IQR) & $21(19-23)$ & $20.8(18.5-22.5)$ & $20.3(19.3-22.3)$ & 0.50 & 0.72 \\
\hline Ethnicity (Caucasian), n (\%) & $20(9 \mid \%)$ & 18 (82\%) & $20(91 \%)$ & 0.58 & 0.78 \\
\hline Age at menarche, in years, median (IQR) & $13(13-14)$ & $13.5(\mid 1-14)$ & $14(12-14)$ & 0.88 & 0.84 \\
\hline Exercise per week, in hours, median (IQR) & $10(8-14)$ & $10(8-14)$ & $13(10-16)$ & 0.60 & 0.07 \\
\hline Type of physical activity-aerobic, n (\%) & $16(73 \%)$ & $18(82 \%)$ & I8 (82\%) & 0.33 & 0.38 \\
\hline Weight loss at time of diagnosis (in $\mathrm{kg}$ ), median (IQR) & $2.25(1.6-3.6)$ & $2.7(2.3-3.5)$ & $2.25(1.7-3)$ & 0.17 & 0.42 \\
\hline
\end{tabular}

Notes: FHA-EX+HA: FHA as well as clinical or biochemical hyperandrogenism; FHA-EX: FHA due to exercise; FHA-AN: FHA due to anorexia nervosa $* P$-value significant at $<0.05$. Abbreviations: FHA, functional hypothalamic amenorrhea; IQR, interquartile range; BMI, body mass index.

at the time of diagnosis was not different between the groups (FHA-EX+HA 2.25 (1.58-3.6) vs FHA-EX 2.7 (2.25-3.49) vs FHA-AN 2.25 (1.69-2.8); $P=0.17$ and $P=0.42$ respectively. There was no difference among the groups based on type of activity (aerobic versus anaerobic) or number of hours of exercise per week (Table 1).

\section{Reproductive phenotype}

There was no difference among the groups based on duration of amenorrhea (Table 2). Clinical signs of hyperandrogenism such as hirsutism were present in $54.5 \%$ of FHA-EX+HA subjects while none of the subjects with FHA-EX and FHA-AN had hirsutism $(P<0.01$ for both). PCOM was present in $95 \%$ of FHA-EX+HA group as compared to $17.7 \%$ of FHA-EX and $0 \%$ in the FHA-AN group ( $P<0.01$ for both). Based on Rotterdam criteria, all subjects in the FHA-EX+HA group met criteria for PCOS.
Mean LH/FSH ratio was higher in FHA-EX+HA than both other groups $(P<0.001)$ (Figure 2$)$. Estradiol concentrations were not available in most subjects, precluding analysis. Progesterone withdrawal was performed in 16/22 of FHA-EX+HA and 22/22 of FHA-EX as well as 17/22 of FHA-AN with percentage of girls failing to menstruate after this being 37.5\% for FHA-EX+HA, 45.5\% for FHA-EX, and $82.4 \%$ for FHA-AN. Estradiol concentrations did not differ between the subsets who failed to menstruate in the FHAEX+HA and FHA-EX groups. Total testosterone concentrations were available in 20 subjects with FHA-EX+HA, 12 subjects with FHA-EX, and eleven subjects with FHA-AN. Total testosterone concentrations were higher in the FHAEX+HA group as compared to FHA-EX $(P=0.04)$ but did not differ from FHA-AN $(P=0.06)$ (Table 2). Dehydroepiandrostenedione sulfate (DHEA-S), free testosterone, and androstenedione concentrations were not available in most

Table 2 Comparison of reproductive, metabolic, and skeletal phenotypes between FHA-EX+HA, FHA-EX, and FHA-AN

\begin{tabular}{|c|c|c|c|c|c|}
\hline Characteristic & $\begin{array}{l}\text { FHA-EX+HA } \\
(n=22)\end{array}$ & $\begin{array}{l}\text { FHA-EX } \\
(n=22)\end{array}$ & $\begin{array}{l}\text { FHA-AN } \\
(n=22)\end{array}$ & $\begin{array}{l}\text { P-value } \\
\text { FHA-EX+HA } \\
\text { vs FHA-EX }\end{array}$ & $\begin{array}{l}\text { P-value } \\
\text { FHA-EX+HA } \\
\text { vs FHA-AN }\end{array}$ \\
\hline \multicolumn{6}{|l|}{ Reproductive phenotype } \\
\hline Duration of amenorrhea (months), median (IQR) & $6(6-11.25)$ & $7(6-12)$ & $6(6-12)$ & 0.55 & 0.68 \\
\hline LH/FSH ratio, median (IQR) & $\mathrm{I} .44(\mathrm{I} .03-\mathrm{I} .77)$ & $0.50(0.20-0.94)$ & $0.67(0.5 \mathrm{I}-0.87)$ & $<0.01$ & $<0.01$ \\
\hline Total testosterone $(\mathrm{ng} / \mathrm{dL})^{*}$, median (IQR) & $31.5(19.8-55.8)$ & $20.5(18-39.8)$ & $23.5(15.5-37)$ & 0.12 & 0.20 \\
\hline Polycystic ovarian morphology (PCOM) (\%) & 95 & 17.7 & 0 & $<\mathbf{0 . 0}$ I & $<\mathbf{0 . 0 1}$ \\
\hline \multicolumn{6}{|l|}{ Metabolic phenotype } \\
\hline Systolic blood pressure (mmHg), median (IQR) & $109(94-116.5)$ & $106(100.8-110.3)$ & $102(94-108)$ & 0.45 & 0.03 \\
\hline Diastolic blood pressure (mmHg), median (IQR) & $69(60-74)$ & $62(54-68.5)$ & $63(56-68)$ & 0.03 & 0.04 \\
\hline Fasting glucose (mg/dL), median (IQR) & $88.5(82.8-90)$ & $83.5(78.8-86.3)$ & 86.5 (83-89) & 0.01 & 0.31 \\
\hline Total cholesterol** (mg/dL), median (IQR) & $183(150.5-232)$ & I57.5 (|43.3-22|.75) & $153(\mid 42-230)$ & 0.44 & 0.62 \\
\hline \multicolumn{6}{|l|}{ Skeletal phenotype } \\
\hline Number of stress fractures, $\mathrm{n}(\%)$ & I (4.5) & $6(27)$ & I I (50) & 0.04 & 0.01 \\
\hline $\begin{array}{l}\text { Bone mineral density (z scores) (spine), }{ }^{* * *} \\
\text { median (IQR) }\end{array}$ & $-0.2(-0.8-0.8)$ & $-I .5(-2.2-1.0)$ & $-1.5(-1.6--0.13)$ & 0.02 & 0.16 \\
\hline
\end{tabular}

Notes: FHA-EX+HA: FHA as well as clinical or biochemical hyperandrogenism; FHA-EX: FHA due to exercise; FHA-AN: FHA due to anorexia nervosa. *Total testosterone concentrations were available in 20 subjects with FHA-EX+HA, 12 subjects with FHA-EX and eleven subjects with FHA-AN. **Total cholesterol was available in I $3 \mathrm{FHA}-\mathrm{EX}+\mathrm{HA}$, ten FHA-EX, and eight FHA-AN subjects. ***DXA z scores were available in four FHA-EX+HA, I4 FHA-EX, and six FHA-AN subjects. Bold denotes $P$-value significant at $<0.05$. Abbreviations: FHA, functional hypothalamic amenorrhea; IQR, interquartile range; BMI, body mass index; vs, versus; LH/FSH, luteinizing hormone/follicle stimulating hormone; DXA, duel-energy X-ray absorptiometry. 

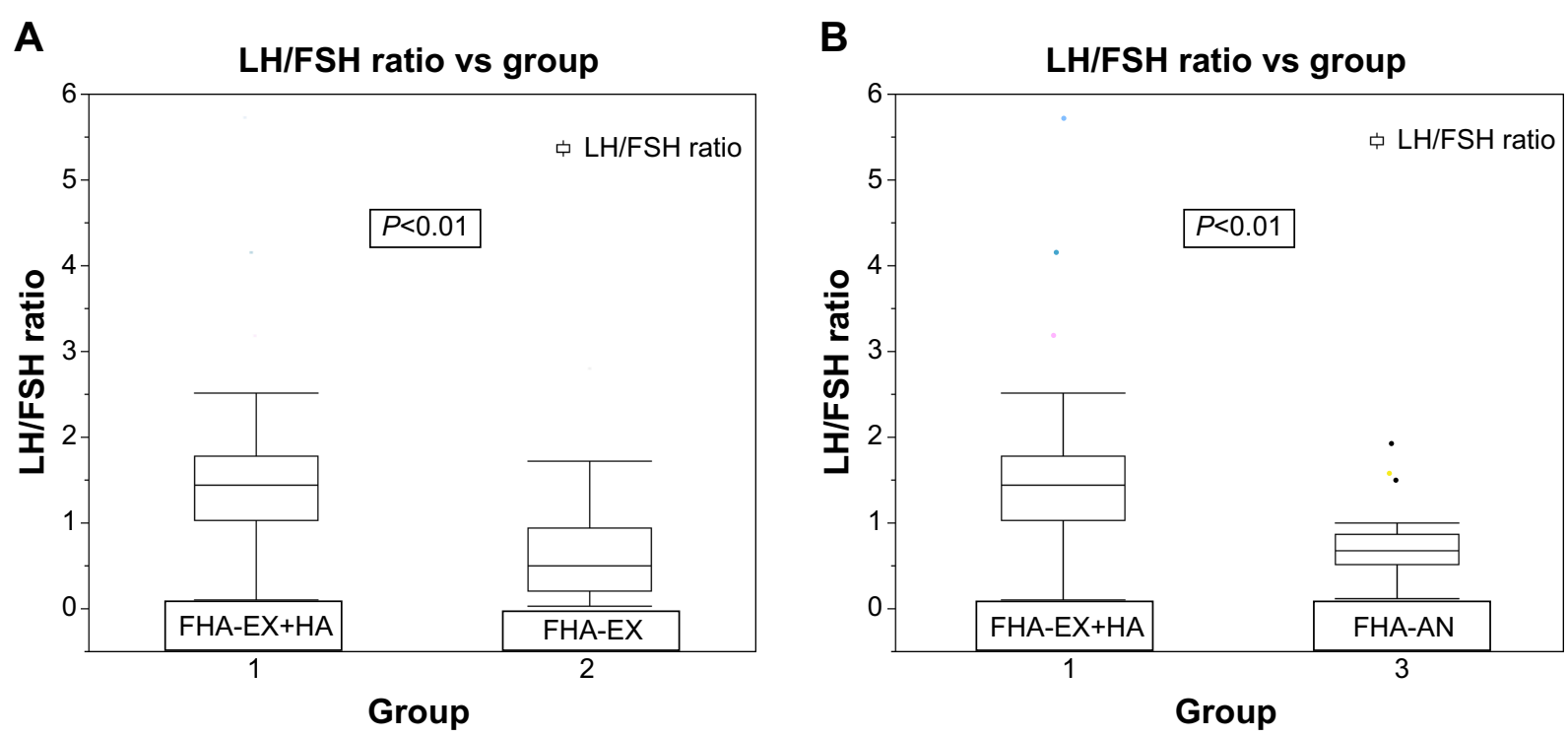

Figure 2 Comparison of LH/FSH ratio between FHA-EX+HA and FHA-EX and FHA-AN.

Notes: (A) Comparison of LH/FSH ratio between FHA-EX+HA and FHA-EX. (B) Comparison of LH/FSH ratio between FHA-EX+HA and FHA-AN. FHA-EX+HA: FHA as well as clinical or biochemical hyperandrogenism; FHA-EX: FHA due to exercise; FHA-AN: FHA due to anorexia nervosa.

Abbreviations: FHA, functional hypothalamic amenorrhea; LH/FSH, luteinizing hormone/follicle stimulating hormone; vs, versus.

subjects, precluding analysis. Pelvic ultrasound evaluation was completed at the time of diagnosis in 20 subjects in the FHA-EX+HA group, 17 subjects in FHA-EX group and 19 FHA-AN subjects.

\section{Metabolic phenotype}

FHA-EX+HA group had higher diastolic blood pressure (DBP) compared to FHA-EX group and higher systolic blood pressure and DBP than FHA-AN group (all $P$-values $<0.05$ ) (Table 2). Mean fasting serum glucose was higher in FHAEX+HA as compared to FHA-EX $(P=0.01)$ but did not differ from FHA-AN group $(P=0.31)$. Total cholesterol concentrations were available for 14 subjects in the FHA-EX+HA group, nine in the FHA-EX group, and eight in the FHA-AN group. There was no difference between the groups based on mean total cholesterol concentrations (Table 2).

\section{Skeletal phenotype}

Percentage of subjects with stress fractures was lower in FHA-EX+HA (4.5\%) as compared to both FHA-EX $(27.3 \%)$ and FHA-AN (50\%) groups $(P=0.04$ and 0.01 respectively). DXA scores at the spine were available for four FHA-EX+HA, 14 FHA-EX, and six FHA-AN subjects. Mean $z$ scores were higher in FHA-EX+HA as compared to FHA-EX ( $P=0.02)$ but did not differ from FHA-AN group (Table 2). Total alkaline phosphatase, calcium, phosphorus, and 25 hydroxy vitamin D concentrations were not obtained in most subjects, precluding analysis.

\section{Relationship between LH/FSH ratio and metabolic phenotype}

There was a positive association between LH/FSH ratio and serum glucose in the entire cohort $(P<0.01)$ (Figure 3 ). This relationship persisted even after adjusting for BMI $(P=0.01)$. When assessed separately in the groups, the relationship was significant only in the FHA-EX+HA cohort $(P=0.04)$, even after adjustment for BMI $(P=0.04)$. There was a positive association between the LH/FSH ratio and DBP $(P=0.01)$, persisting after adjustment for BMI $(P=0.02)$. No association was present between the LH/ FSH ratio and systolic blood pressure or total cholesterol (all $P$-values $>0.05$ ).

\section{Relationship between $\mathrm{LH} / \mathrm{FSH}$ ratio and skeletal phenotype}

There was no association between the LH/FSH ratio and the number of stress fractures.

However, there was a positive association between $\mathrm{LH} /$ FSH ratio and DXA spine score in the entire cohort $(P=0.04)$, attenuated after adjusting for BMI $(P=0.09)$ (Figure 3).

\section{Discussion}

Although reproductive dysfunction in female athletes is typically ascribed to energy deficit and inhibition of gonadotropin-releasing hormone pulsatility, ${ }^{25}$ our study highlights the unique reproductive phenotype, with higher $\mathrm{LH} / \mathrm{FSH}$ ratios of athletes with FHA due to excessive 

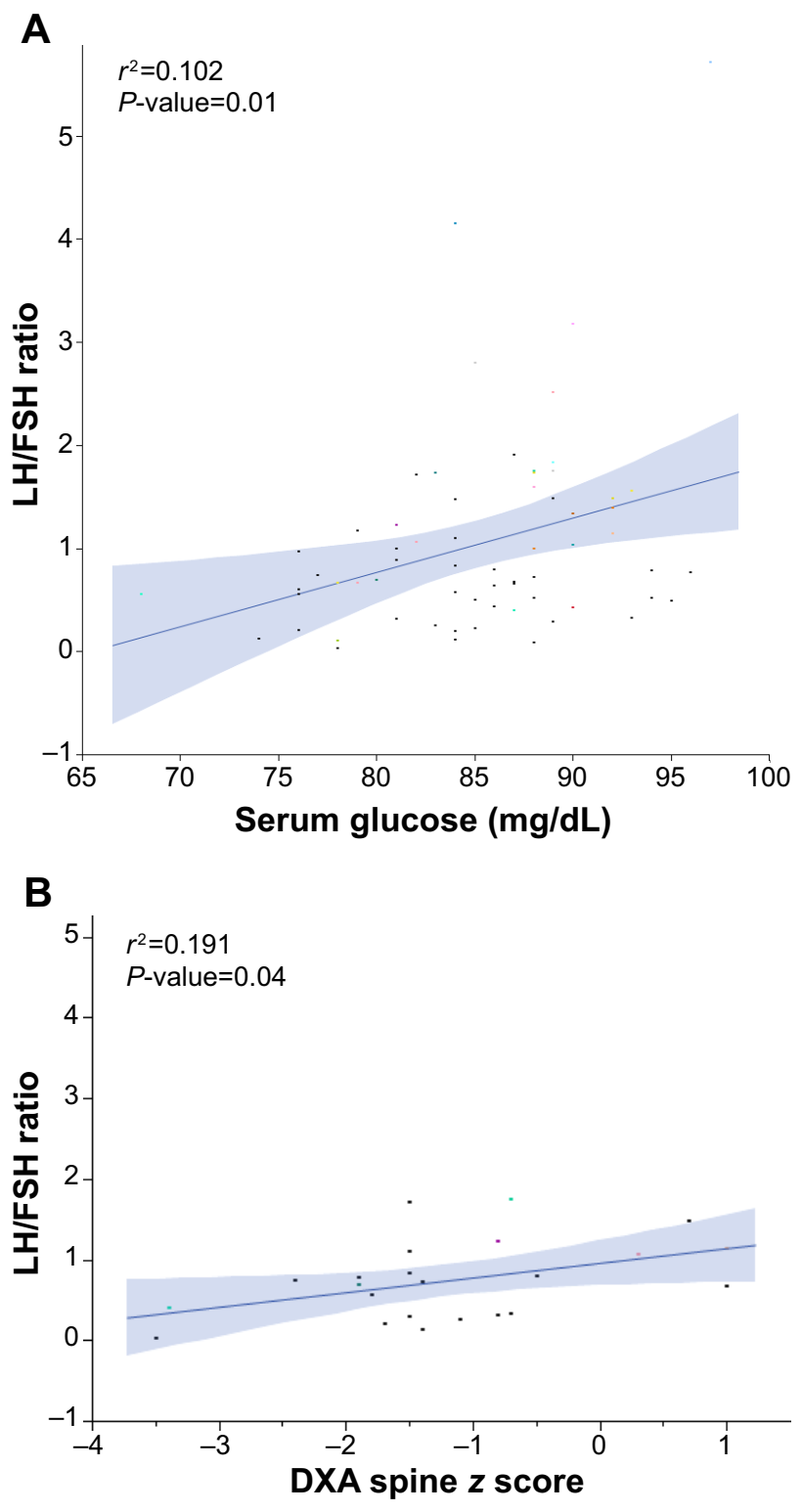

Figure 3 Linear regression models depicting relationship between LH/FSH ratio and serum glucose $(\mathrm{mg} / \mathrm{dL})(\mathbf{A})$ and relationship between LH/FSH ratio and DXA spine $z$ score $(B)$.

Abbreviations: $\mathrm{LH} / \mathrm{FSH}$, luteinizing hormone/follicle stimulating hormone; DXA, duel-energy $\mathrm{X}$-ray absorptiometry.

exercise who also have evidence of clinical or biochemical hyperandrogenism as compared to BMI matched females with FHA due to exercise or anorexia nervosa alone. We also report a possible adverse metabolic profile with higher fasting glucose in these athletes with hyperandrogenism as well as an improved bone mineral density resulting in fewer stress fractures in this cohort.

Rickenlund et al describe the existence of hyperandrogenism in a subset of endurance athletes with menstrual dysfunction with elevated LH/FSH ratios and androgen concentrations. ${ }^{22}$ In a previous study of 90 Swedish Olympic athletes, the most common cause of menstrual dysfunction was PCOS and not hypothalamic inhibition ${ }^{26}$ but although previous studies have reported existence of a subset of athletes with menstrual dysfunction attributable, at least in part, to hyperandrogenism, ${ }^{22,26}$ our study is the first to our knowledge to compare the reproductive, metabolic, and skeletal health parameters among females with both menstrual dysfunction and hyperandrogenism (FHA-EX+HA) to BMI matched groups of females with FHA due to exercise or anorexia nervosa alone. In addition to a higher $\mathrm{LH} / \mathrm{FH}$ ratio in the subset of athletic women with hyperandrogenism, we also report higher blood pressure and fasting glucose in this group as compared to BMI matched subjects with FHA due to excessive exercise or anorexia nervosa alone. The association of LH/FSH ratio with fasting glucose has not previously been reported in young female athletes with menstrual dysfunction and hyperandrogenism. In subjects with PCOS, and nationally representative women without PCOS, a distinct subset with excess LH and hyperinsulinemia has been reported. These women typically have higher adrenal androgenic activity and markers of chronic inflammation and dyslipidemia which have been shown to be significantly associated with LH/FSH ratio. ${ }^{27,28}$ To further confirm this association in our cohort, this association of LH/FSH ratio with fasting glucose persisted only in the subgroup of athletic women with hyperandrogenism (FHA-EX+HA).

Although a previous small series has reported an association between improved bone mineral density and hyperandrogenism in female athletes ${ }^{22}$ and in lean women with PCOS,${ }^{29}$ the findings of our study are novel in the sense of reporting a positive association between LH/FSH ratio and stress fractures as well as bone mineral density (BMD) DXA $z$ scores at the spine in athletes with and without evidence of hyperandrogenism as well as another comparator group of females with anorexia nervosa. Although causality is difficult to establish in a retrospective study, the LH/FSH ratio may well serve as a marker of metabolic and skeletal health in young athletic females. Therefore, it may serve not just as a diagnostic tool but also indicate when further screening for metabolic risk factors may be warranted. An important clinical implication of our study findings pertains to management of these females. While the existing treatment focuses on energy repletion above all else, one may have to exercise some caution prior to recommending significant increase in caloric intake or weight in this subset of hyperandrogenic young women as their metabolic profiles may be adversely impacted. In the FHA-EX+HA group, a significant number of women had withdrawal bleeding after progesterone 
challenge, serving as further evidence that menstrual dysfunction in this subset may arise from excessive androgen concentration or activity rather than hypoestrogenism.

\section{Strengths}

The strengths of our study include the comparison of athletic young women with menstrual dysfunction along with clinical or biochemical hyperandrogenism with two comparator groups ie, women with FHA due to excessive exercise (FHA-EX) or anorexia nervosa (FHA-AN). Also, exclusion of subjects with potential disordered eating from $\mathrm{FHA}-\mathrm{EX}+\mathrm{HA}$ and FHA-EX was performed after extensive review of clinical charts. Further, the matching of BMI between groups allowed us to negate the impact of weight associated changes on metabolic and skeletal health parameters. Lastly, unlike prior investigations on the subject, we excluded women who were on any hormonal medications at the time of diagnosis in order to not impact the gonadotropin or androgen concentrations.

\section{Limitations}

There is a potential risk of selection bias conducting a retrospective observational study that selects groups from different specialties. Also, despite careful clinical review, it is possible that some subjects in the FHA-EX+HA and FHA-EX groups had some disordered eating while others meeting criteria for anorexia nervosa also had significant exercise habits but not documented in charts. Similarly, the distinction between amenorrhea secondary to FHA or hyperandrogenism may be quite difficult as we relied on medical record diagnosis of FHA along with evidence of hyperandrogenism to define the group FHA-EX+HA. We hope, however, that comparison to two groups with documented diagnoses of FHA due to exercise or anorexia nervosa alone with no clinical or biochemical evidence of hyperandrogenism would further support the conclusions reported. Additionally, missing or limited data for some parameters is also a challenge of retrospective data collection. Similarly, we relied solely on medical records to define the group FHA-EX+HA as athletes with FHA along with evidence of androgen excess, making the distinction between amenorrhea secondary to FHA or to FHA with hyperandrogenism difficult. We also acknowledge that in 2006, there was a change in the LH assay but this did not affect reference ranges and a validation study showed good correlation between the two immunoassays. Future prospective studies systematically studying the hormonal profile of young athletic women and body composition as well as markers of metabolic function are needed to further elucidate any underlying relationships.

\section{Conclusion}

Young athletic women may suffer from menstrual dysfunction due to reasons other than energy depletion and FHA. In the subset of young active women with clinical or biochemical hyperandrogenism, higher LH/FSH ratios than similar aged women with comparable weight who suffer from FHA due to excessive exercise or anorexia nervosa can be seen. This can serve as an important diagnostic indicator and have clinical implications on management of this subset of young women since indiscriminate caloric repletion and weight gain may adversely impact their metabolic profile. Large, prospective studies are needed to delineate the relationship between these phenotypes in athletic women.

\section{Disclosure}

The authors report no conflicts of interest in this work.

\section{References}

1. Pinhas-Hamiel O, Pilpel N, Carel C, Singer S. Clinical and laboratory characteristics of adolescents with both polycystic ovary disease and anorexia nervosa. Fertil Steril. 2006;85(6):1849-1851.

2. Golden NH, Carlson JL. The pathophysiology of amenorrhea in the adolescent. Ann N Y Acad Sci. 2008;1135:163-178.

3. Gordon CM. Clinical practice. Functional hypothalamic amenorrhea. N Engl J Med. 2010;363(4):365-371.

4. Liu JH, Bill AH. Stress-associated or functional hypothalamic amenorrhea in the adolescent. Ann N Y Acad Sci. 2008;1135:179-184.

5. Legro RS, Lin HM, Demers LM, Lloyd T. Rapid maturation of the reproductive axis during perimenarche independent of body composition. J Clin Endocrinol Metab. 2000;85(3):1021-1025.

6. Baumann EE, Rosenfield RL. Polycystic ovary syndrome in adolescence. The Endocrinologist. 2002;12:333-348.

7. Knochenhauer ES, Key TJ, Kahsar-Miller M, Waggoner W, Boots LR, Azziz R. Prevalence of the polycystic ovary syndrome in unselected black and white women of the southeastern United States: a prospective study. J Clin Endocrinol Metab. 1998;83(9):3078-3082.

8. Dunaif A, Thomas A. Current concepts in the polycystic ovary syndrome. Annu Rev Med. 2000;52:401-419.

9. Azziz R, Woods KS, Reyna R, Key TJ, Knochenhauer ES, Yildiz BO. The prevalence and features of the polycystic ovary syndrome in an unselected population. J Clin Endocrinol Metab. 2004;89(6): 2745-2749.

10. Gabrielli L, Aquino EM. Polycystic ovary syndrome in Salvador, Brazil: a prevalence study in primary healthcare. Reprod Biol Endocrinol. 2012;10:96.

11. Azziz R, Carmina E, Dewailly D, et al. Position statement: criteria for defining polycystic ovary syndrome as a predominantly hyperandrogenic syndrome: an Androgen Excess Society guideline. J Clin Endocrinol Metab. 2006;91(11):4237-4245.

12. Zawadzki JK, Dunaif A. Diagnostic criteria for polycystic ovary syndrome: towards a rational approach. In: Dunaif A, Givens JR, Haseltine F, Merriam G, editors. Polycystic Ovary Syndrome. Cambridge, MA: Blackwell Scientific; 1992:377-384.

13. Azziz R, Carmina E, Dewailly D, et al. The Androgen Excess and PCOS Society criteria for the polycystic ovary syndrome: the complete task force report. Fertil Steril. 2009;91(2):456-488.

14. Centers for Disease Control and Prevention [homepage on the Internet]. CDC Growth Charts [updated September 9, 2010]. Available from: http:// www.cdc.gov/growthcharts/cdc_charts.htm. Accessed November 10, 2014. 
15. DeUgarte CM, Bartolucci AA, Azziz R. Prevalence of insulin resistance in the polycystic ovary syndrome using the homeostasis model assessment. Fertil Steril. 2005;83(5):1454-1460.

16. Mathur R, Alexander CJ, Yano J, Trivax B, Azziz R. Use of metformin in polycystic ovary syndrome. Am J Obstet Gynecol. 2008; 199(6):596-609.

17. Palmert MR, Gordon CM, Kartashov AI, Legro RS, Emans SJ, Dunaif A. Screening for abnormal glucose tolerance in adolescents with polycystic ovary syndrome. J Clin Endocrinol Metab. 2002;87(3):1017-1023.

18. Sam S, Dunaif A. Polycystic ovary syndrome: syndrome XX? Trends Endocrinol Metab. 2003;14(8):365-370.

19. Coviello AD, Legro RS, Dunaif A. Adolescent girls with polycystic ovary syndrome have an increased risk of the metabolic syndrome associated with increasing androgen levels independent of obesity and insulin resistance. J Clin Endocrinol Metab. 2006;91(2):492-497.

20. Constantini NW, Warren MP. Menstrual dysfunction in swimmers: a distinct entity. J Clin Endocrinol Metab. 1995;80(9):2740-2744.

21. Warren MP, Perlroth NE. The effects of intense exercise on the female reproductive system. J Endocrinol. 2001;170(1):3-11.

22. Rickenlund A, Carlstrom K, Ekblom B, Brismar TB, von Schoultz B, Hirschberg AL. Hyperandrogenicity is an alternative mechanism underlying oligomenorrhea or amenorrhea in female athletes and may improve physical performance. Fertil Steril. 2003;79(4):947-955.

23. Alsara A, Warner DO, Li G, Herasevich V, Gajic O, Kor DJ. Derivation and validation of automated electronic search strategies to identify pertinent risk factors for postoperative acute lung injury. Mayo Clin Proc. 2011;86(5):382-388.
24. Rotterdam ESHRE/ASRM-Sponsored PCOS Consensus Workshop Group. Revised 2003 consensus on diagnostic criteria and longterm health risks related to polycystic ovary syndrome. Fertil Steril. 2004;81(1):19-25.

25. Javed A, Tebben PJ, Fischer PR, Lteif AN. Female athlete triad and its components: toward improved screening and management. Mayo Clin Proc. 2013;88(9):996-1009.

26. Hagmar M, Berglund B, Brismar K, Hirschberg AL. Hyperandrogenism may explain reproductive dysfunction in olympic athletes. Med Sci Sports Exerc. 2009;41(6):1241-1248.

27. Banaszewska B, Spaczynski RZ, Pelesz M, Pawelczyk L. Incidence of elevated $\mathrm{LH} / \mathrm{FSH}$ ratio in polycystic ovary syndrome women with normo- and hyperinsulinemia. Rocz Akad Med Bialymst. 2003;48:131-134

28. Beydoun HA, Beydoun MA, Wiggins N, Stadtmauer L. Relationship of obesity-related disturbances with LH/FSH ratio among post-menopausal women in the United States. Maturitas. 2012;71(1):55-61.

29. Zborowski JV, Cauley JA, Talbott EO, Guzick DS, Winters SJ. Clinical Review 116: Bone mineral density, androgens, and the polycystic ovary: the complex and controversial issue of androgenic influence in female bone. J Clin Endocrinol Metab. 2000;85(10):3496-3506.
International Journal of Women's Health

\section{Publish your work in this journal}

The International Journal of Women's Health is an international, peerreviewed open-access journal publishing original research, reports, editorials, reviews and commentaries on all aspects of women's healthcare including gynecology, obstetrics, and breast cancer. The manuscript management system is completely online and includes

\section{Dovepress}

a very quick and fair peer-review system, which is all easy to use. Visit http://www.dovepress.com/testimonials.php to read real quotes from published authors. 